\title{
Review of Literature Update Index, A New Index For Peer Reviewed Articles
}

\author{
Hussein G El Charkawi* \\ Professor and Chairman of Prosthodontics Department, Faculty of Oral and Dental Medicine, Future University, Cairo, Egypt
}

Received: 眥: November 27, 2018; Published: 制: December 05, 2018

*Corresponding author: Hussein G El Charkawi, Professor of Prosthodontics, Faculty of Oral and Dental Medicine, Future University, Cairo, Egypt

\begin{abstract}
This article presented a new and simple numerical index to help peer reviewers of scientific articles in assessment of the updated references and knowledge used in a given article. The Charkawi Update Index CUI index proposed in this article is based on giving a score out of ten that indicate the number of recent publications in the last 5 or 10 years. The CUI-index aims to maintain high levels of quality, improve processing and provide reliability. It also, aims to decrease the amount of subjectivity in the judgment process of peer reviewing. This Index (CUI) could be applied to any article, review papers, case reports, books and even research projects. The CUI-index provided a simple, yet indicative and accurate way in assessment of how original and timely the article being reviewed. It also, helps in detection of any irrelevant and weak evidences in the discussion section of the reviewed articles.
\end{abstract}

\section{Introduction}

Designing the future system, by which we evaluate papers and decide which ones deserve broad attention and deep reading, is a great challenge of our time. The reviewer' job as a journal advisor is to make sure that the best possible research appears in print. The purpose of peer review is to ensure

a. High standard, checking that no mistakes in procedure or logic have been made;

b. That the results presented support the conclusion drawn;

c. That no mistakes in citations to previous work have been made;

d. That all human and animal protocols conducted follow proper review and approval by appropriate Institutional Review Committees; and, very importantly,

e. That the work is original and relevant [1].

\section{History}

Since the first prototype professional peer-review process was started in the ninth century in the Ethics of the Physician written by Ishaq bin Ali al-Rahwi (854-931). His work indicates that a visiting physician must make duplicate notes of a patient's condition on every visit. When the patient healed or had died, the notes of the physician were examined by a local medical council of other physicians, who would decide whether the treatment had met the required standards of medical service [2]. Peer review is the evaluation of work by one or more people of similar proficiency to the producers of the work (peers). It constitutes a form of selforganization by qualified members of a profession within the relevant field. Peer review methods are employed to maintain high levels of quality, improve processing, and provide reliability. In academia peer review is often used to rule out an academic paper's appropriateness for publication.

\section{Problem}

As peer reviews process has many merits, it has also, some limitations. Yoshitaka Fujii currently holds the record for the most fraudulent articles of the scientific community. The Japanese anesthesiologist, who faked 172 papers, was dismissed by the University of Toho for lacking proper ethical approval for clinical studies in several of his papers. It later emerged that there was no evidence Fujii had collected any of the data published in his later retracted articles. Like many cases of fraud, this scenario raises questions about how misconduct went undetected for so long. In a world of peer review and hard data, how can fraudulent cases of such epic proportion be possible? And potential fraud is not the only concern; peer review is supposedly a central pillar of modern science, but a growing number of scientists are speaking out about what they regard to be unacceptable flaws in the system $[2,3]$. One of the major problems that confront the process of reviewing articles is to keep it as quality-control system for published articles 
and concurrent to be far apart from being a subjective process of evaluation that have bias and errors [4].

Unless universal standards are formulated, manuscript reviews remain subjective, imperfect and inconsistent at best. The time has come to reconsider the parameters of this system. This article aims to set a standard criterion in evaluation of Review of literature updates that help reviewers in the assessment of the reviewed articles by a numerical scoring system that indicates the overall originality and latest in cutting edge research of the paper submitted. The date of the reference is very important. Some topics, such as those in the health-related sciences, require contemporary information. Other subjects, such as Geology, value older material as well as current. However, Knowing the time $\mathrm{n}$ frame of the topic and examine the timeliness of the article is totally subjective and rating such; up-to-date, out-of-date, or timeless cannot help the reviewer to achieve a proper evaluation.

\section{Charkawi Updates Index CUI}

This Index will target peer review of research manuscripts submitted to scientific journals, but many of the elements of peer review can be applied to other areas, such as grants and books. The review section should include up to date references and be based on as wide and thorough a search of sources as possible. This simple score depend on counting the references included in the published article within the last five years from the publication acceptance date. Not only have that but included also, the evaluation of updated references in the discussion section and it shows to the reviewers how much the authors validated their results according to the most updated knowledge in the field examined.

If the total count of the references published in the last five years was

a. $100 \%$ this equal to score $10 / 10$

b. $75 \%$ this equal to score $9 / 10$

c. $50 \%$ this equal to score $8 / 10$

d. $25 \%$ this equal to score $7 / 10$

e. $15 \%$ this equal to score $6 / 10$

f. $10 \%$ this equal to score $5 / 10$

g. $5 \%$ this equal to score $4 / 10$

h. Less than $5 \%$ this equal to score $2 / 10$

A slight modification could be made especially in review papers that are considered millstones and bench mark in literature review and as it should cover the history and origin of the tested idea. This is achieved by counting references that were published in the last 10 years back from appearance of the article:
a. $100 \%$ this equal to score $9 / 10$
b. $75 \%$ this equal to score $8 / 10$
c. $50 \%$ this equal to score $7 / 10$
d. $25 \%$ this equal to score $6 / 10$
e. $15 \%$ this equal to score $5 / 10$
f. $10 \%$ this equal to score $4 / 10$
g. $5 \%$ this equal to score $3 / 10$
h. Less than $5 \%$ this equal to score $1 / 10$

This numerical system was based on that usually any article could have only few updated references and almost never happened that an article was $100 \%$ updated. An article could be rated updated if it is more than $50 \%$ and moderately updated if it is more than $25 \%$ and poorly updated if less than $15 \%$. This Index (CUI) could be applied to review papers, case reports and even research projects. The Impact Factor (IF) which is a measure reflecting the average number of citations to recent articles published in the journal. However, all journals have a profusion of citations, and even the best have some deceitful papers and some miserably defective ones. So, it is ludicrous to judge an individual paper solely on the IF of the journal in which it is published [5]. The Eigenfactor (EF), on the other hand scores are measures of a journal's importance and overall value provided by all of the articles published in a given journal in a year. It is apparent that no single guide sufficiently quantifies the creativeness of an article [5]. Both IF and EF can be used in combination with CUI-index to evaluate the work of individual This article in the discussion section and to detect any weak logic or irrelevant evidence.

\section{Benefits}

There is a strong consensus that the peer review process should include written reviews and numerical ratings. These classic fundamentals of peer review continue to be useful. The CUI index has many advantages. It gives the reviewers a numerical tool that helps them in assessment of the originality of the paper submitted, review coverage to all aspects of the subject and meanwhile to evaluate objectively the validity of the research to the current knowledge in the field. However, it helps reviewers to avoid largely speculative criticisms not supported by specific data or literature; or lack of evidence that the criticisms are actually relevant to the specific data in the paper [6-8]. It also, reflects high ethical standards for both the reviewer and the author/s. This index helps the reviewer to detect fraud as well as any weak and unsupported logic especially in the discussion section. A true example of weak evidences just occurred. I was reviewing an article that is supposed to appear in January 2014 about hollow implants. The author usedas an evidence to support his logic for using the hollow implant- an article that was published in 1988. The author did not recognize that during this last 26 years, the hollow implant is no longer in use, and showed many problems such as bone saucerization, implant mobility or continuous pain in the implant area. Histology in many cases indicated a progressive bone resorption that extended down to the hollow basket area of the implant [9].

This implant does not even manufactured anymore! Because I have applied the CUI-index in this particular case, I was able to identify why the logic that the author depending on is completely 
irrelevant, unjustified and unacceptable. In the last eight articles that I have reviewed, it was found that CUI index was as follow (Table 1). In the other hand, CUI-index encourages authors to review the recent research papers in their domain and incorporated them in their articles. It provides a strong incentive for authors to heed the advice and to improve the paper [8]. As a reviewer to many scientific journals, I have implemented this index for many years during the process of reviewing articles and generated this numerical score that helped the overall evaluation process. I have found that this index is beneficial and decrease the amount of subjectivity in the judgment process. Kriegeskorte et al. [8] emphasized that the most important traits for a reviewer to have are courtesy, fairness, and punctuality [8]. However, this index arguably may also, provide a definitive signal of a paper's de-facto importance.

Table 1: CUI index.

\begin{tabular}{|c|c|c|c|}
\hline Article \# & Number of Ipdated References & Percentage & Score \\
\hline 1 & $5 / 24$ & $20.8 \%$ & $6 / 10$ \\
\hline 2 & $7 / 23$ & $31 \%$ & $7 / 10$ \\
\hline 3 & $14 / 23$ & $60.8 \%$ & $8 / 10$ \\
\hline 4 & $15 / 23$ & $65.2 \%$ & $8 / 10$ \\
\hline 5 & $1 / 21$ & $4.7 \%$ & $2 / 10$ \\
\hline 6 & $8 / 30$ & $26.6 \%$ & $7 / 10$ \\
\hline 7 & $8 / 35$ & $22.8 \%$ & $6 / 10$ \\
\hline 8 & $9 / 27$ & $30 \%$ & $7 / 10$ \\
\hline
\end{tabular}

\section{Limitations}

The proposed index although indicative; it is still cover only single facet of the peer review process. However, further attempts

ISSN: 2574-1241

DOI: 10.26717/BJSTR.2018.11.002150

Hussein G El Charkawi. Biomed J Sci \& Tech Res

This work is licensed under Creative

Commons Attribution 4.0 License

Submission Link: https://biomedres.us/submit-manuscript.php in transferring the peer review process to an objective qualitycontrol method should be given ways to appear.

\section{Conclusion}

The CUI- index proposed in this article that depends on counting the references cited in a certain article in the last five year before publication provided a simple, yet efficient and accurate way in assessment of how original and timely the article being reviewed.

\section{References}

1. Benos DJ, Bashari Edlira, Chaves JM, Gaggar A, Kapoor N, et al. (2007) The Ups and Downs of Peer Review. Advances in Physiology Education 31(2): 145-152.

2. Spier Ray (2002) The history of the peer-review process. Trends in Biotechnology 20(8): 357-358.

3. Natalie Healey (2013) The problem with peer review. Laboratory News.

4. Couzin Frankel J (2013) Secretive and Subjective, Peer Review Proves Resistant to Study. Science 341(6152): 1331.

5. Fresh A (2009) The most influential journals: Impact Factor and Eigenfactor Proc Natl Acad Sci U S A, 106(17): 6883-6884.

6. Thomas Wesley Allen (2013) Peer Review Guidance: How Do You Write a Good Review? J Am Osteopath Assoc 113(12):916-920.

7. Jennings C (2006) Quality and Value: the true purpose of peer review. Nature.

8. Kriegeskorte N, Walter A, Deca Diana (2012) An emerging consensus for open evaluation: 18 visions for the future of scientific publishing. Frontiers in Computational Neuroscience 6(1): 94.

9. Albrektsson TB Becker, K Higuchi, P Krogh, J Malmquist, Sennerby L (1992) Failure of core-vent implants: A retrieval analysis of 19 hollow basket implants. Materials 10(4): 219-224.

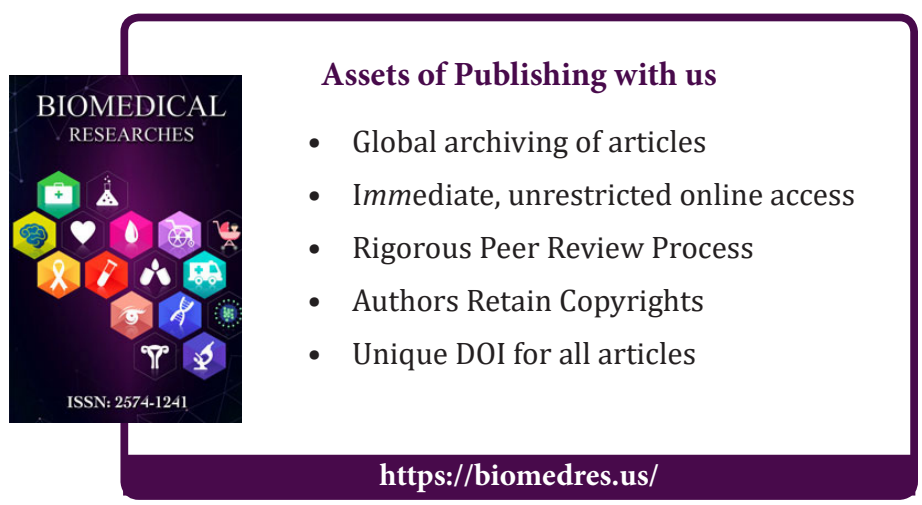

